\title{
A comparative analysis of Heat Shock Factor 1 in Cancer
}

Shouhartha Choudhury ( $\nabla$ shouharthac@gmail.com )

Assam University

\section{Research Article}

Keywords: HSF1, HSF family, Gene Therapy, Cancer Immunotherapy

Posted Date: August 25th, 2021

DOI: https://doi.org/10.21203/rs.3.rs-820519/v1

License: (c) (i) This work is licensed under a Creative Commons Attribution 4.0 International License. Read Full License 


\section{Abstract}

The heat shock factor 1 (HSF1) is a DNA-binding factor that has responses under proteotoxic stress is well-known as an HSF. Heat shock factor 1 is a unique regulator that coordinates chaperone expression to enhance organism growth. Specifically, HSF's drive transcriptional strategy is apparent from the HSP's and promotes cell viability. A recent study supported the HSF1 coordinated with different cancer and robust in cancer immunotherapy. In this research, I designed to genome-wide analysis of HSF1 and its family in mammals. A comparative analysis of HSF's is mandatory to explore functions and immunogenic mechanisms involved with HSP's in organisms. Therefore, I performed a bioinformatics pipeline and tools to the current knowledge of HSF's in two different organisms. This application can enhance in a significant way to study particular HSF's in discrete organisms. My finding data demonstrated the number of HSF1 and their family in Homo sapiens and Mus musculus. The conserved domain, motifs, phylogeny, chromosome location, gene network, and expression analysis documented the HSF1 associated with cancer. The analysis data concluded the HSF1 is impact in various cancers and a signature of cancer immunotherapy.

\section{Introduction:}

The expensive investigation in biology is how cells suddenly adapt to the affected environment. The massive environmental stress can damage cells. The multicellular organisms have a response to generate HSPs (heat-shock proteins) or chaperones to repair protein homeostasis [1-6]. This robust mechanism is well-known as an HSR (heat shock reaction) and releases HSF1. That HSF1 phosphorylate, trimerize and relocate to the nucleus. Also, HSF1 involved with chaperone protein expression by bounding to DNA sequence motifs is well-known as HSE's. It becomes clear the organisms share molecular responses that include the dramatic transformation of gene expression and synthesis stress induce protein [7-10]. The molecular study suggested HSR represents unique examples of stress-inducible gene expression impart by the transcription factor is called HSF. The multi-cellular organisms have a reaction of heat stress by bounding the initial transcriptional catalyst to DNA. The HSF1 is preserved in evolution also act as stress tolerance to activate HSP. A protective factor conserved over species. The HSF1 is a leading catalyst of protein folding with defensive activities is generally known as HSP's (chaperone protein). The HSPs such as chaperonin 10 (HSPE1 or HSP10), chaperone DnaJ (HSP40), TCP-1/cpn60 (HSP60), DnaK (HSP70), HSP90, HSP90B1 (Grp94/Gp96), HSP110, and Glucose-regulated protein 170 (Grp170) retain intercellular homeostasis for protecting the proteome against the toxic reactions of protein folding, misfolding and accumulation. HSP's provoke immunity that offers a unique advantage for cancer immunotherapy. The activity of HSP's helps to define the immune reaction that produces by the cells in our body under a stable or stressful environment. HSP's protect the cell's stress, such as unstable temperature, oxidative element, heavy metals and lower $\mathrm{pH}$ [11-13]. The immune cells define peptides and epitopes on surface antigens, HSPs enhance molecular fragments included both peptides and epitopes on cells. The HSP-base immunity is vital to elicit memory T-cell and antigen-specific cytotoxic lymphocytes response. The polypeptides bind with HSP's to activate acquired immunity 
associated antigens by the process of dendritic cells. The antigen produces when the multiple proteases react in the proteasome. Then the antigens appear on the cell face by the functions of MHC1 that elevate in cells and process CD8 + T-cells to allow immunologic surveillance. During antigen cross-presentation, exogenous antigenic attributes are eliminated by lysosomes and forwarded on the cell periphery by activation of $\mathrm{MHC} 2$ that moderate the immune process. It assumed that different antigenic activity allows external antigens to penetrate $\mathrm{MHC} 1$. The antigen cross-presentation empowers surface antigens to appear dendritic cells by mighty functions of $\mathrm{MHC1}$ and activate $\mathrm{CD} 8+\mathrm{T}$-cells to kill malignant cells [14-18]. The cross-presentation of antigens elevates two alternative mechanisms: (a) the Cytosolic pathway of antigen cross-presentation and (b) TAP (TAP1/TAP2) [19]. Besides, initiation of MHC1/MHC2 sustains cell surface via non-specific endocytosis on cell surface receptors. Assessment of antigen governs the antigen cross-presentation that implicates Fc receptors, CLEC, CLEC9A, CD209 (DC-SIGN), CD205 (DEC205) and CD206 [20-23]. Hypothetically, HSP's bound to SRs such as SREC-1/SCARF1, FEEL-1 (STAB1), LOX-1 (OLR1), CD204, and CD91 (LRP1). However, HSP70 bind with large granular lymphocytes (LGL), CD314 (NKG2D) and OLR1 belong to the SRs and CLEC (C-type lectin) [24-26, 12]. The SREC-1 receptors bind with DnaK, HSP90, HSPH1, HSP90B1 and GRP170 to appear antigen receptors in DC. The maturation of endosome and endosome-plasma membrane to cytosol-cell membrane requires HSP70 and HSP90 mediated antigen cross-presentation [27-29]. HSP base peptides increase antigens by proteolysis through cell compartments and access antigens to initiate cytotoxic Tcell cross-priming. The cells activate immunity when MHC1 interact with CTL by process of APC and epitopes cross-presentation. An encounter of chaperones included DnaK, HSP90, Grp78 (HSPA5 or Byun1) and Grp94 (gp96 or ERp99) suspected immune functions. It is unclear that Grp78 (HSPA5) is bound with peptides and participates in antigen allowance but has an immune reaction in chaperoning of $\mathrm{IgG}$. The HSP70 release from cells and aspect necrosis lead to a robust T-cell mediated anti-tumour immune response. Also, GRP170 produce under the extracellular environment acquires tumour rejection via effects of LGL even cytotoxic T-cell. The silencing of exosomes effect on DC contains HSP70 in crosspriming of T-cells potentially executed a role of immunity. However, tumour specific exosomes embrace DnaK on the vesicle surface and appear immunosuppression via interaction of MDSC [30-35]. Cross presentation of HSP-based peptide-binding antigens is not sufficient to complete the program of CTL without other molecular negotiation and may fail to proliferate and encounter PCD. In cytotoxic T-cell response, DC process antigens via MHC2 and activate $\mathrm{CD} 4+\mathrm{T}$-cells. It presumes that HSPs chaperone antigens activate through the process of MHC2 and initiate CD4 + T-cells. The stimulating of CD8 + T-cell requires a DC permit and negotiation with CD4 + T-cells [36-37]. Preliminary interaction of CD154 (CD40L) on CD4 + T-cell surface by process of CD40 on DC signals initiate co-stimulatory molecules CD80 (B7-1) and CD86 (B7-2) both permitted CD8 + T-cells activity. Also, PD-L1 and PD-L2 maintain costimulatory function via the reaction of PD-1 (CD279). The T-cell surface molecules CD28 and CTLA-4 (CD152) interacts with co-stimulatory ligands B7-1 and B7-2 on APCs and regulated T-cell antigen recognition. Co-stimulatory molecules rewarded by CD28 function on T-cells and support signals of CD8 + T-cells by the process of MHC1-peptide-complex on T-cell receptor. During illness, PAMPs bound to the PRR also TLR and stimulate innate immune reactions via APC. That type of signal activates nuclear factor-KB that evokes proinflammatory cytokine and co-stimulatory molecules such as B7-1 and B7-2 
[38-44]. Furthermore, HSP's base peptides potentially functioned as DAMPs induce innate immunity via interaction of TLR2 and TLR4 dependant fashion. The SREC-1 dependent tumour immunity activate by HSP70 require TLR signalling through MYD88 or TLR2/TLR4 receptors to destroy tumours. As CTL activation, the participation of DC, CD $8+T$-cells, and CD $4+T$-cells all are sharply regulate by the process of inflammatory cytokines via DC. Remarkably, HSP70 activates by the activity of CCL cytokines in TLR4 dependent fashion and bind directly to the CCR5 [45-47]. Also, LOX-1 even SREC-1 interacts with TLR2 to activate innate immunity through HSP associated LOX-1 signalling. The fold of LOX-1 with rectifying LDL produce MAP kinase, NF-KB and pro-inflammatory signalling through the intracellular domain receptors. HSP's interact with MHC1 via SRA/CD204 receptors to innate immune signalling through interactions of TLR4, MAP kinase, and NFKB that innate CD4 + T-cell activation. HSP90 dependent SREC-1 integrates GTPase and non-receptor tyrosine kinase strongly associated with lipid rafts in TLR4 signalling manner and innate-immune response. HSP stimulate signalling of SREC1 also LOX-1 represent antigen crosspresentation even stimulates innate immunity and control the inhibitory response of HSP dependent SRA/CD204 signalling. The HSP's base peptide-binding suggested the combined action of SREC1 and LOX-1 enhances functional response on the cell surface. The significant prototype of tumour evolves HSP peptide expect an ability to bind antigenic oligopeptides. Therefore, the HSP's associated peptide binding is relatively stable when ADP rather than ATP is adjacent to the chaperone $[12,14,28,44]$. This immunologic theorem extended for chaperone properties atop and their respective abundance inside the cells and supported in different cancer. Moreover, HSF's have multiple isoforms acquire in organisms, which appear discrete functions like physiological distress, oogenesis, early development, embryonic growth and various diseases in mammals. One of them is HSF1 provoke rapidly in a hypoxic and hostile microenvironment that is a growth inhibitor in an individual. The cancer cells express high levels of the HSF's to protect the folding of malformed proteins and solubilization [48-55]. The HSF1 in cancer is a target impact on malignancy also activates a broad array of malignant cells, and then the template of DNA tenancy is divergent from the normal cells. Also, HSF1 govern sophisticated transcriptional activity that promotes cellular transformation and sustains malignant growths. Those concepts suggested HSF's prominently associated with different cancer. Therefore, classical mechanisms of HSF's maintain cell cycle regulation, cell signalling, metabolism, adhesion and translation [1]. In this study, I decided to a comparative analysis of the HSF1 and their family in mammals. The assessment of the HSF family is a monument for future examination of the particular HSF in different organisms.

\section{Results:}

\section{Structural analysis}

The target (query) sequence determined the precise formation of nucleotide and peptide. The sequence composed of 1590 nucleotides and 529 peptides within 105 peptides bind to the DNA sequence is known as an HSF domain (Table. 1). The polypeptide structure demonstrated that the HSF domain bind to the HSE's. The HSE's are a palindromic sequence with repeated pyrimidine and purine motifs. The HSF1 is homotrimeric cytoplasmic polypeptide results of relocalisation of the nucleus. The HSF that monomer contains $\mathrm{C}$-terminal also N-terminal leucine zipper replicates. The sequence of HSF1 bound with N- 
terminal zipper-fit unity of two-dimensional nuclear localisation signals. Interaction of $\mathrm{N}$ terminal also $\mathrm{C}$ terminal-zipper result of structural mask in nuclear localisation, the activation of HSF1 is an un-maske result of relocalisation protein in the nucleus. The DNA binding components recline in the $\mathrm{N}$ terminus for nuclear localisation signals (Fig. 1).

\section{Genome-wide analysis}

The genome-wide analysis by the HMMER algorithm obtains the multiple hits 28 and 23 of HSF domains in Homo sapiens and Mus musculus, respectively (Table 2a). The standalone BLAST result represents 14 and 25 homologs in Homo sapiens and Mus musculus, respectively (Table 2a). The multiple hits listed from both organisms for annotation, the gene ontology annotation demonstrated the sequence accuracy of the particular genes in the HSF family (Table 3). A total of 5 and 9 of HSF1 and 28 and 25 of HSF's validate in Homo sapiens and Mus musculus, respectively (Table 2b).

\section{Domain, motifs, and phylogeny analysis}

The highest hits of HSF1 listed from both organisms for sequence aligning, an MSA result determines the conserved HSF domain. The high consensus (90\%) indicates the extended HSF domain (Fig. 2) and motifs (Fig. 3). The phylogenetic tree demonstrated the molecular evolutionary link of the HSF1 in between Homo sapiens and Mus musculus. Particular clade represents the multifunctional heat shock factors in both organisms (Fig. 4).

\section{Expression, chromosome location, and network analysis}

The disease state study suggested the HSF1 express in the breast, intraductal carcinoma, neoplasms of digestive organs, colon, carcinoma, neoplasms of respiratory system/intrathoracic organs, bronchus, lung, non-small cell carcinoma, squamous cell carcinoma, and adenocarcinoma (Fig. 5, 6, 7). The chromosome location study confirms the HSF1 located band 8q24.3, start 144,291,591 bp and ends $144,314,720$ bp (Fig. 8). The gene network study determines the HSF1 gene interacts with other molecules, such as HSP90AB1, HSPA1A, HSP90AA1, SIRT1, MAPK3, MAPK1, EEF1A1, HSPA8, DNAJB1, and HSBP1, those molecular interactions govern the outcome of the HSF1 in the cells (Fig. 9).

\section{Discussion:}

A comparative analysis of the HSF1 and their family regulated malignancy also the HS (heat shock) TF's (transcription factors) govern HSP's complex in oncogenesis. HSF1 activate in a large diversity of human malignancies and is strongly correlated in different cancer worldwide [53]. The broad array of tumours suggested the HSF1 rival an inherent character in tumour biology. Many concepts arise during heat shock activation i.e. protein folding, ATP formation, composite of extracellular matrix, DNA repair, translation, cell signalling, cellular adhesion and apoptosis affected by the HSF's. The remarkable hypothesis in this research is an outcome of heat shock factor 1 is a mediator of HSP chaperone activities [1]. The response of HSF1 in cancer explains: (a) why HSF1 is dynamic modifiers in tumorigenesis (b) why HSF1 determine 
metastasis-promoting gene (c) what activation clearly define query gene as a regulator in cancer [54]. Many years of investigation have not rigorously elucidated the HSF1 process under classical heat shock reaction. The functional mechanisms demonstrated the cascade of the target gene during segregation by functions of chaperones when unravel substrates engage with chaperone binding. HSF1 response in malignancy during protein homeostasis, misfolding oncoproteins, and aneuploidy. The increased rates of abnormal cells activity significantly stimulate HSF1 reaction and release chaperones. The unstable signalling in abnormal cells drives post-transcriptional modification; those mechanisms suggested HSF1 is a subject of extensive post-transcriptional modification, acetylation, and phosphorylation [55]. Also, the action of metastasis and death is a conserved origin explicit from cancer. A broad parameter of tumours with HSF1 activity supported the fundamental biological process. The response of HSF1 regulated high growth also metabolism as a wound healing and immune reaction [1]. HSF1 also HSF3 function as stress-responsive activator regulates heat-shock reaction, whereas HSF2 activated during outgrowth and segregation. HSF4 is express in the brain, heart, skeletal muscle and pancreas. Also, HSF5 regulates for recovery of the morphological changes of growth-arrested by apoptosis. The X/Y-linked HSF's are a testis-specific factor initiate's antibody. Paradoxically, the evolutionary functions of HSF1 in cells adapt, survive, propagate, and support in cancers. HSF1 cumulated oncogenic mutation for disrupting physiology and metastasis [56-58]. The diagnosis value of heat factors is a diverse regulator that activates variable targets for cancer immunotherapy. This study demonstrated the fundamental treatment benefit of HSF1 in various cancers. The clinical implementation must require for the achievement of signature and validation of samples in multiple clinical cohorts. The potential establishment of HSF1 supported the breakthrough of lethal tumours. Therefore, my consciousness of the immense role of HSF's supported in different cancers in humans.

\section{Material And Methods:}

\section{Database and target sequence}

The primary structure retrieves from various specific databases such as UniProt, NCBI, KEGG, DDBJ and EMBL. Then we perform web-based application SMART for examinations of the particular domain in the query sequence. Pfam search for retrieving protein family details. SWISS-MODEL performs for constructing a three-dimensional structure of the objective sequence. SWISS-MODEL is a bioinformatics web-server for comparative modelling of 3D structures. Also, this application offers to generate a viable structure for practical works. That application makes an experimental protein to rebuild the structure for evolutionary proteins. This database is up to date for comparative or homology modelling of proteome for biomedical research.

\section{Genome}

The draft genome sequences all organisms download from various databases such as Ensemble and NCBI.

\section{Standalone tools}


HMMER executes through MSA of peptide residues as an initial search. HMMER is a mathematical and statistical algorithm that offers to makes an MSA of the peptide domain as a primary search. An implemented method that uses probabilistic models is well-known as profile HMM. Standalone BLAST2 conducts for the homologs in isolated organisms.

\section{Gene annotation}

The BLAST2GO performed for gene ontology annotation is bioinformatics and a mathematical tool for high-throughput gene annotation of the high-resolution sequences. The functional sophistication of the particular genes retrieves via the GO (gene ontology) database is a standard vocabulary of the working attribute.

\section{Domain}

For observation of a conserved domain, an MSA method is applicable to calculate the accurate match of the homologs sequences. So the identity, similarity, and differences can be measure. MSA of multiple hits sequences from selected organisms carried by web-based application MultAlin.

\section{Motif}

The MEME application performs for observation of sequence motifs. That is a computational web base tool to analyze even discovery of the sequence motifs.

\section{Chromosome location}

Chromosome location retrieves using a database gene card. The gene card database of an organism's gene content provides knowledge of all human genes and their chromosome location. This database is currently available for biological research like genes, proteins, and their associated diseases.

\section{Gene networks}

The genetic (gene) network is a group of molecules that regulate and interact in the cells to control the degree of mRNA or proteins expression. Some proteins convey to activate genes called TF's that bind to the promoter area and initiate the formation of other proteins is called regulatory cascades. We can retrieve the STRING database for the prediction of protein-protein interaction. The STRING database contains various resources like experimental data and computational methods.

\section{Declarations}

The work furnished in this paper is original and communicated by the correspondent placed in the manuscript. The author disclosed that the document is not concern elsewhere and not receive for evaluation by other journals.

\section{Ethical agreement:}


The study contains an in-silico analysis of the mammalian genome to validate the particular TF's gene in different organisms.

\section{Availability of supplementary data and material:}

The data and samples may be available on request or demand.

\section{Conflict of interest:}

The author declared that the work has no conflict of interest.

\section{Funding:}

The author did not avail of financial assistance from any source in undertaking the present study.

\section{Acknowledgement:}

The author is thankful to Assam University, Silchar, Assam, India, for arranging the lab space during this research work.

\section{Abbreviations}

HSF: Heat Shock Factor

HSE: Heat Shock Element

HSP: Heat Shock Protein

HSF1: Heat Shock Factor 1

HSF2: Heat Shock Factor 2

HSF3: Heat Shock Factor 3

HSF4: Heat Shock Factor 4

HSF5: Heat Shock Factor 5

TAP: Transporter associated with antigen processing

HSFX: Heat Shock Factor X-linked

HSFY: Heat Shock Factor Y-linked

CTL: Cytotoxic Lymphocyte

APC: Antigen Presenting Cell 
MHC1: Major Histocompatibility Class 1

MHC2: Major Histocompatibility Class 2

CD8+ T-cell: Cytotoxic T-cell

CLEC: C-type lectins

DCs: Dendritic cells

CLEC9A: C-type lectin domain family 9 member A

DC-SIGN: Dendritic Cell-Specific Intercellular adhesion molecule-3-Grabbing Non-integrin

CD209: Cluster of Differentiation 209

DEC205: Lymphocyte antigen 75 or CD205

CD206 (Cluster of Differentiation 206): Mannose Receptor 1

SR: Scavenger Receptor

SCARF1: Scavenger Receptor Class F Member 1

FEEL-1 (STAB1): Stabilin-1

LOX-1 (OLR1): Oxidized low-density lipoprotein receptor 1 (lectin-type oxidized LDL receptor 1)

SRA/CD204: Macrophage scavenger receptor 1

LRP1 (CD91): Low density lipoprotein receptor-related protein 1

NK: Natural Killer

NKG2D (CD314): Killer Cell Lectin-like Receptor K1

CLEC: C-type Lectin Families

SREC-1: Scavenger Receptor Class F Member 1

SRs: scavenger receptors

HSP70 (DnaK): Heat shock protein 70 (70 kilodalton heat shock proteins)

HSP90: Heat shock protein 90

HSP40: Heat shock protein 40 (chaperone DnaJ) 
HSP60: Heat shock protein 60

HSP90B1: Heat shock protein 90 kDa beta member 1 (endoplasmin, gp96, grp94, or ERp99)

HSP110: Heat shock protein 110

HSP10: Heat shock 10 kDa protein 1 (chaperonin 10, cpn10 or early-pregnancy factor)

Grp78 (HSPA5 or Byun1): Binding immunoglobulin protein (BiP)

Grp94 (gp96 or ERp99): Heat shock protein 90kDa beta member 1 (HSP90B1) or endoplasmin IgG: Immunoglobulin G

Grp170: Glucose-regulated protein 170

MDSC: Myeloid-derived suppressor cells

PCD: Programmed cell death

CD4+ Cells: T helper cells

CD80 (B7-1): Cluster of differentiation 80

CD86 (B7-2): Cluster of Differentiation 86

B7-DC (CD273): Programmed cell death 1 ligand 2

B7-H1 (CD274): Programmed death-ligand 1

PD1 (CD279): Programmed cell death protein 1

CD28: Cluster of Differentiation 28

CTLA-4 (CD152): Cytotoxic T-lymphocyte-associated protein 4

PAMPs: Pathogen-associated molecular patterns

PRR: pattern recognition receptors

TLR: Toll-like receptor

NFkB: Nuclear factor-kB

DAMPs: Damage-associated molecular patterns

TLR2: Toll-like receptor 2 
TLR4: Toll-like receptor 4

CCL: Chemokine receptors

CCR5: C-C chemokine receptor type 5

LDL: Low-density lipoprotein

MAPK: Mitogen-activated protein kinase

CDC42: Cell division control protein 42 homolog

ADP: Adenosine diphosphate

ATP: Adenosine triphosphate

DNA: Deoxyribonucleic acid

MYD88: Myeloid differentiation primary response 88 (innate immune signal transduction adaptor)

\section{References}

1.Mendillo, M.L., HSF1 drives a transcriptional program distinct from heat shock to support highly malignant human cancers. Cell, 2012. 150(3): p. 549-562.

2.Scherz-Shouval, R., The reprogramming of tumor stroma by HSF1 is a potent enabler of malignancy. Cell, 2014. 158(3): p. 564-578.

3.Vabulas, R.M., Protein folding in the cytoplasm and the heat shock response. Cold Spring Harbor perspectives in biology, 2010. 2(12): p. 004390.

4.Shamovsky, I. and E. Nudler, New insights into the mechanism of heat shock response activation. Cellular and Molecular Life Sciences, 2008. 65(6): p. 855-861.

5.Habib, H.N. and W.M.M. Saleh, The Role of Heat Shock Proteins 70 (HSP70) in Farm Animals Adaptation, A Review Paper.

6.Gökmen-Polar, Y. and S. Badve, Upregulation of HSF1 in estrogen receptor positive breast cancer. Oncotarget, 2016. 7(51): p. 84239.

7.Kihara, F., Heat shock factor binds to heat shock elements upstream of heat shock protein 70a and Samui genes to confer transcriptional activity in Bombyx mori diapause eggs exposed to $5 \mathrm{C}$. Insect biochemistry and molecular biology, 2011. 41(11): p. 843-851. 
8.Sakurai, H. and Y. Enoki, Novel aspects of heat shock factors: DNA recognition, chromatin modulation and gene expression. The FEBS journal, 2010. 277(20): p. 4140-4149.

9. Lindquist, S. and E. Craig, The heat-shock proteins. Annual review of genetics, 1988. 22(1): p. 631-677.

10. Georgopoulos, C., R.I. Morimoto, and A. Tissières, Stress proteins in biology and medicine. 1990: Cold Spring Harbor Laboratory.

11. Wu, C., Heat shock transcription factors: structure and regulation. Annual review of cell and developmental biology, 1995. 11(1): p. 441-469.

12. Ayesha Murshid, Jianlin Gong, Mary Ann Stevenson and Sturat K Calderwood et al, Heat shock proteins and cancer vaccines: developments in the past decade and chaperoning in the decade to come. Expert Review of Vaccines, 2011. 10(11): p. 1553-1568

13. R Suto and PK Srivastava et al, A mechanism for the specific immunogenicity of heat shock proteinchaperoned peptides. Science, 1995. 269(5230): p. 1585-1588

14. Ayesha Murhid, Jianlin Gong and Stuart K. Calderwood et al, The role of heat shock proteins in antigen cross presentation. Front. Immunol, 2015. 3(63)

15. William R Heath and Francis R Carbone, Dendritic cell subsets in primary and secondary $T$ cell responses at body surface. Nature Immunology, 2009. 10: p. 1237-1244

16.JJ Neefjes, F Momburg and GJ Hammerling at al, Selective and ATP-dependent translocation of peptides by the MHC-encoded transporter. Science, 1993. 261(5122): p. 769-771

17. Christopher C. Norbury, Sameh Basta, Keri B. Donohue, David C. Tscharke, Michael F. Princiotta and Peter Berg et al, CD8+ T Cell Cross-Priming via Transfer of Proteasome Substrates. Science, 2004. 304(5675): p. 1318-1321

18. Paul G. Thomas1 and Peter C. Doherty et al, New Approaches in Immunotherapy. Science, 2010. 327(5963): p. 249

19. Kenneth L. Rock and Lianjun Shen et al, Cross presentation: underlying mechanisms and role in immune surveillance. Immunological Reviews, 2005. 27(1): p. 166-183

20. Laura Bonifaz, David Bonnyay, Karsten Mahnke, Miguel Rivera, Michel C. Nussenzweig, and Ralph M. Steinman et al, Efficient Targeting of Protein Antigen to the Dendritic Cell Receptor DEC-205 in the Steady State Leads to Antigen Presentation on Majo Histocompatibility Complex Class I Products and Peripheral CD8+ T Cell Tolerance. J Exp Med, 2002. 196(12): p. 1627-1638

21. Sven Burgdorf, Veronika Lukacs-Kornek and Christian Kurts et al, The Mannose Receptor Mediates Uptake of Soluble but Not of Cell-Associated Antigen for Cross-Presentation. J Immunol, 2006. 176(11): 
p. $6770-6776$.

22. Juliana Idoyaga, Ashira Lubkin, Christopher Fiorese, Mireille H. Lahoud, Irina Caminschi, Yaoxing Huang, Anthony Rodriguez, Björn E. Clausen, Chae Gyu Park, Christine Trumpfheller, and Ralph M. Steinman et al, Comparable T helper 1 (Th1) and CD8 T-cell immunity by targeting HIV gag p24 to CD8 dendritic cells within antibodies to Langerin, DEC205, and Clec9A. PNAS, 2011. 108(6): p. 2384-2389

23. Paul J. Tacken, Wiebke Ginter, Luciana Berod, Luis J. Cruz, Ben Joosten, Tim Sparwasser, Carl G. Figdor, Alessandra Cambi et al, Targeting DC-SIGN via its neck region leads to prolonged antigen residence in early endosomes, delayed lysosomal degradation, and cross-presentation. Blood, 2011. 118 (15): p. 4111-4119.

24. Robert J. Binder, David K. Han and Pramod K. Srivastava et al, CD91: a receptor for heat shock protein gp96. Nature Immunology, 2000. 1: p. 151-155.

25. Yves Delneste, Giovanni Magistrelli, Jean-François Gauchat, Jean-François Haeuw, Jean- Pierre Aubry, Kayo Nakamura, Naoko Kawakami-Honda, Liliane Goetsch, Tatsuya Sawamura, Jean-Yves Bonnefoy, and Pascale Jeannin et al, Involvement of LOX-1 in Dendritic Cell-Mediated Antigen Cross-Presentation. Immunity, 2002. 17: p. 353-362.

26. Jimmy R. Thériault, Hideki Adachi and Stuart K. Calderwood et al, Role of Scavenger Receptors in the Binding and Internalization of Heat Shock Protein 70. J Immunol, 2006. 177 (12): p. 8604-8611.

27. Masoud H. Manjili, Robert Henderson, Xiang-Yang Wang, Xing Chen, Ying Li, Elizabeth Repasky, Latif Kazim and John R. Subjeck et al, Development of a Recombinant HSP110-HER-2/neu Vaccine Using the Chaperoning Properties of HSP110. Cancer Research, 2002. 62: p. 1737-1742.

28. Brent Berwin, Yves Delneste, Rachel V. Lovingood, Steven R. Post and Salvatore V. Pizzo et al, SREC-I, a Type F Scavenger Receptor, Is an Endocytic Receptor for Calreticulin*, The Journal of Biological Chemistry, 2004. 279(49): p. 51250-51257.

29. Ayesha Murshid, Jianlin Gong and Stuart K. Calderwood et al, Heat Shock Protein 90 Mediates Efficient Antigen Cross Presentation through the Scavenger Receptor Expressed by Endothelial Cells-I. J Immunol September, 2010. 185 (5): p 2903-2917.

30. Jun Kunisawa and Nilabh Shastri et al, Hsp90aChaperones Large C-TerminallyExtended Proteolytic Intermediatesin the MHC Class I Antigen Processing Pathway. Immunity, 2006. 24: p. 523-534.

31. Monika C. Wolkers, Gerd Stoetter, Florry A. Vyth-Dreese and Ton N. M. Schumacher et al, Redundancy of Direct Priming and Cross-Priming in Tumor-Specific CD8+ T Cell Responses. J Immunol, 2001. 167 (7): p. 3577-3584.

32.Yasunori Kozutsumi, Karl Normington, Eileen Press, Clive Slaughter, Joe Sambrook, Mary Jane Gething et al, Identification of immunoglobulin heavy chain binding protein as glucose-regulated protein 78 on the 
basis of amino acid sequence, immunological cross-reactivity, and functional activity. Journal of Cell Science, 1989. 11 (10): p. 115-137.

33. Gregory A Daniels, Luis Sanchez Perez, Rosa Maria Diaz, Timothy Kottke, Jill Thompson, Maoyi Lai, Michael Gough, Mahzuz Karim, Andrew Bushell, Heung Chong, Alane Melcher, Kevin Harrington and Richard G Vile et al, A simple method to cure established tumors by inflammatory killing of normal cells. Nature Biotechnology, 2004. 22: p. 1125-1132.

34. Julien Taieb, Nathalie Chaput, Noël Schartz, Stéphan Roux, Sophie Novault, Cédric Ménard, François Ghiringhelli, Magali Terme, Antoine F. Carpentier, Guillaume Darrasse-Jèse, François Lemonnier and Laurence Zitvogel et al, Chemoimmunotherapy of Tumors: Cyclophosphamide Synergizes with Exosome Based Vaccines, J Immunol, 2006. 176(5): p 2722- 2729.

35. Fanny Chalmin, Cedric Rebe and Francois Ghiringhelli et al, Membrane-associated Hsp72 from tumorderived exosomes mediates STAT3-dependent immunosuppressive function of mouse and human myeloid-derived suppressor cells. J Clin Invest, 2010. 120 (2): p. 457-471.

36. Guangming Gong, Lingyun Shao,Yunqi Wang, Crystal Y. Chen, Dan Huang, Shuyu Yao, Ximei Zhan, Helene Sicard, Richard Wang and Zheng W. Chen et al, Phosphoantigen- activated Vy2Vס2 T cells antagonize IL-2-induced CD4+CD25+Foxp3+T regulatory cells in mycobacterial infection. Blood, 2009. 113 (4): p. 837-845.

37. By Sally R.M. Bennett, Francis R. Carbone, Freda Karamalis, Jacques F.A.P. Miller, and William R. Heath et al, Induction of a CD8+ Cytotoxic T Lymphocyte Response by Cross-priming Requires Cognate CD4+ T Cell Help. J Exp Med, 1997. 186 (1): p. 65-70.

38. Christian Kurts, Bruce W. S. Robinson and Percy A. Knolle et al, Cross-priming in health and disease. Nature Reviews Immunology, 2010. 10: p. 403-414.

39. Shengdian Wang, Jürgen Bajorath, Dallas B. Flies, Haidong Dong, Tasuku Honjo, and Lieping Chen et al, Molecular Modeling and Functional Mapping of B7-H1 and B7-DC Uncouple Costimulatory Function from PD-1 Interaction, J Exp Med, 2003. 197 (9): p. 1083-1091.

40. Cynthia A Chambers and James Allison et al, Co-stimulation in T cell responses. Current Opinion in Immunology, 1997. 9(3): p. 396-404.

41. By P. Anton van der Merwe, Dale L. Bodian, Susan Daenke, Peter Linsley and Simon J. Davis et al, CD80 (B7-1) Binds Both CD28 and CTLA-4 with a Low Affinity and Very Fast Kinetics. J. Exp. Med., 1997. 185(3): p. 393-403.

42. Masahiro Yamamoto and Kiyoshi Takeda, Current Views of Toll-Like Receptor Signaling Pathways. Gastroenterology Research and Practice, 2010. 2010: 971270. 
43. Anette Gjorloff Wingren, Eduardo Parra, Mikael Varga, Terje Kalland, Hans-Olov Sjogren, Gunnar Hedlund and Mikael Dohlsten et al, T Cell Activation Pathways: B7, LFA-3, and ICAM-1 Shape Unique T Cell Profiles. Critical Reviews in Immunology, 1995. 15(3\&4): p. 235-153.

44. Christropher E. Rudd, Alison Taylor and Helga Schneider, CD28 and CTLA 4 coreceptor expression and signal transduction. Immunological Reviews, 2009. 229(1): p. 12-26.

45. Jianlin Gong, Bangmin Zhu, Ayesha Murshid, Hideki Adachi, Baizheng Song, Allegra Lee, Chunlei Liu and Stuart K. Calderwood et al, T Cell Activation by Heat Shock Protein 70 Vaccine Requires TLR Signaling and Scavenger Receptor Expressed by Endothelial Cells-1. J Immunol, 2009, 183 (5): p. 30923098.

46. Trevor Whittall, Yufei Wang, Justine Younson, Charles Kelly, Lesley Bergmeier, Barry Peters, Mahavir Singh and Thomas Lehner Dr et al, Interaction between the CCR5 chemokine receptors and microbial HSP70. European Journal of Immunology, 2006. 36(9): p. 2304- 2314.

47. Zhiyuan Shen, Tianyi Li, Da Chen, Sen Jia, Xiangming Yang, Liang Liang, Juan Chai, Xiaobing Cheng, Xinjie Yang and Moyi Sun et al, The CCL5/CCR5 axis contributes to the perineural invasion of human salivary adenoid cystic carcinoma. Oncology Reports, 2013. 31(2): p. 800-806.

48. Morimoto, R.I., Regulation of the heat shock transcriptional response: cross talk between a family of heat shock factors, molecular chaperones, and negative regulators. Genes \& development, 1998. 12(24): p. 3788-3796.

49. Sarge, K.D., et al., Cloning and characterization of two mouse heat shock factors with distinct inducible and constitutive DNA-binding ability. Genes \& Development, 1991. 5(10): p. 1902-1911.

50. Schuetz, T.J., et al., Isolation of a cDNA for HSF2: evidence for two heat shock factor genes in humans. Proceedings of the National Academy of Sciences, 1991. 88(16): p. 6911-6915.

51. Xiao, X., et al., HSF1 is required for extra embryonic development, postnatal growth and protection during inflammatory responses in mice. The EMBO journal, 1999. 18(21): p. 5943-5952.

52. Drysdale, M.J., et al., Targeting Hsp90 for the treatment of cancer. Current opinion in drug discovery \& development, 2006. 9(4): p. 483-495.

53. Whitesell, L. and S.L. Lindquist, HSP90 and the chaperoning of cancer. Nature Reviews Cancer, 2005. 5(10): p. 761.

54. Solimini, N.L., J. Luo, and S.J. Elledge, Non-oncogene addiction and the stress phenotype of cancer cells. Cell, 2007. 130(6): p. 986-988.

55. Anckar, J. and L. Sistonen, Regulation of HSF1 function in the heat stress response: implications in aging and disease. Annual review of biochemistry, 2011. 80: p. 1089-1115. 
56. M. Gabriella Santoro et al, Heat Shock Factors and the Control of the Stress Response. Biochemical Pharmacology, 2000. 59: p. 55063

57. Marshad Speevak and Mario Chevrette et al, Human Chromosome 3 Mediates Growth Arrest and Suppression of Apoptosis in Microcell Hybrids. Molecular and Cellular Biology, 1996. 16(5): p. 2214-2225

58. Kalager, M., et al., Prognosis in women with interval breast cancer: population based observational cohort study. Bmj, 2012. 345: p. e7536.

\section{Tables}

Due to technical limitations, Table 1,2 and 3 are only available as a download in the Supplemental Files section.

\section{Figures}

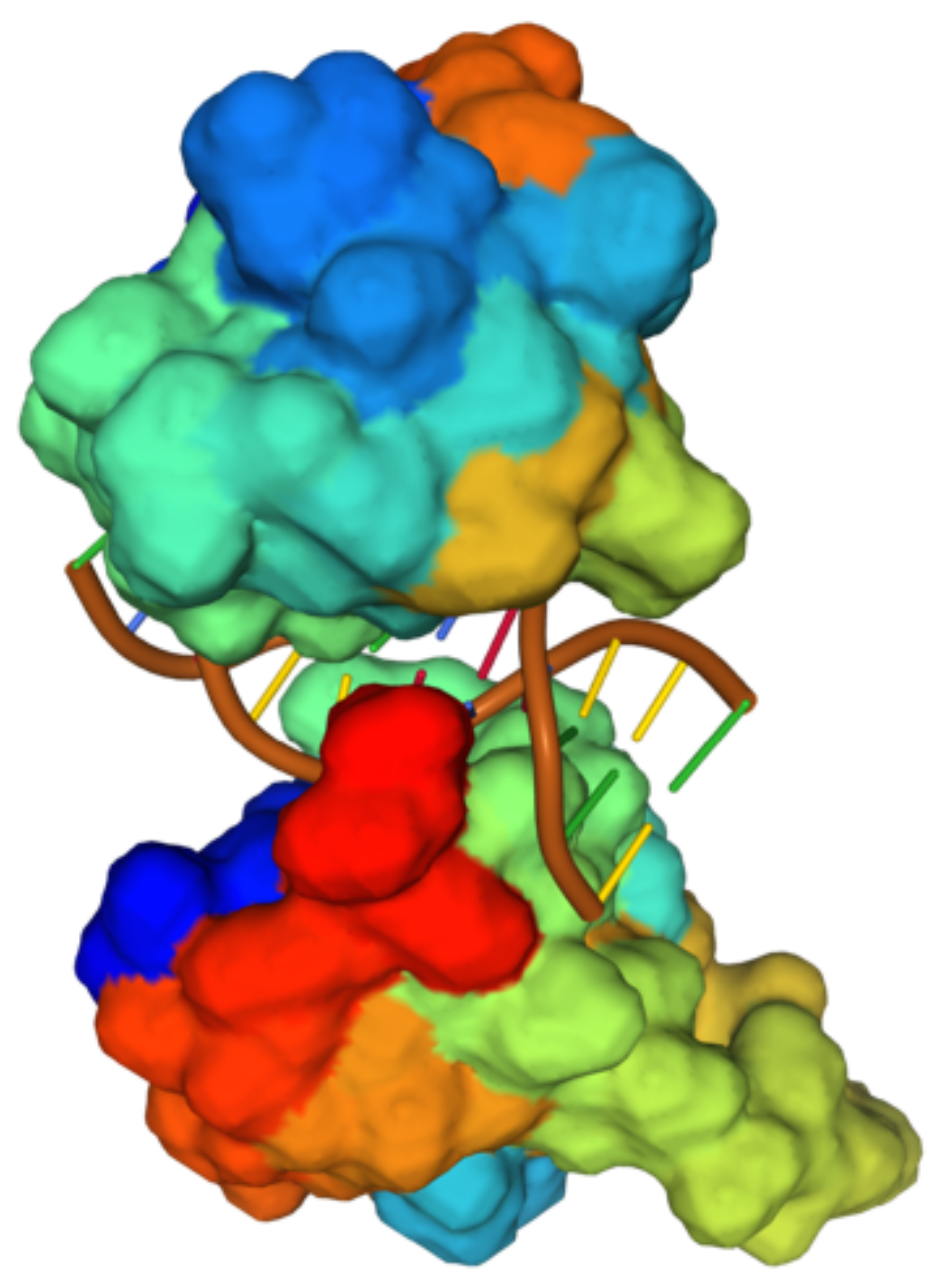

Figure 1 
1

20

30

40

50

60

70

80

90

100

110

120

130

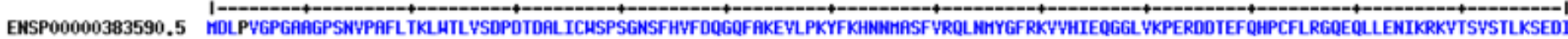

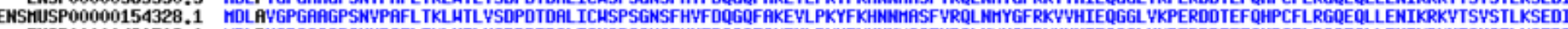

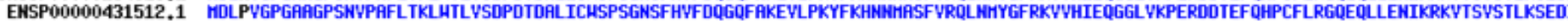

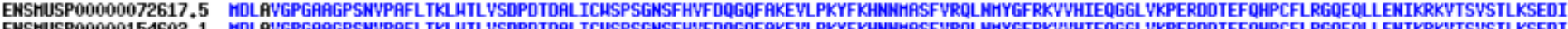
ENSHUSP0000154602.1 MDLAVGPGAAGPSNYPAFLTKLHTLYSDPDTDHLICHSPSGNSFHYFDQGQFAKEVLPKYFKHNHHASFVRQLNHYGFRKVVHIEQGGLVKPERDDTEFQHPCFLRG0EQLLENIKRKVTSVSTLKSEDI ENSHUSP00000154638,1 ENSHUSP00000154386.1 Consensus

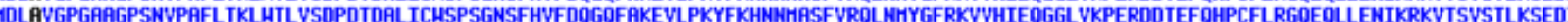

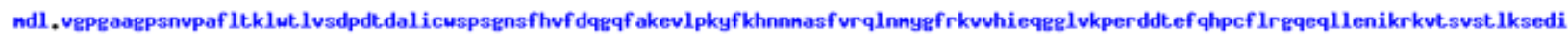

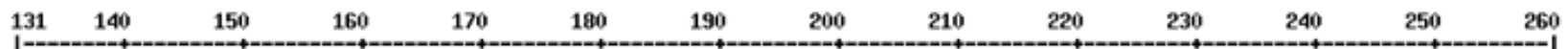

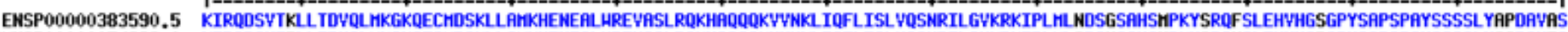

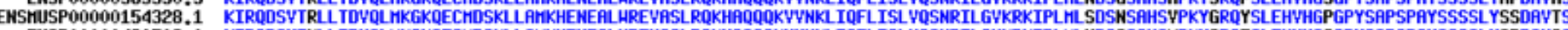

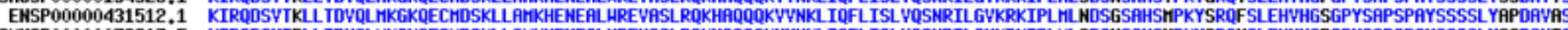

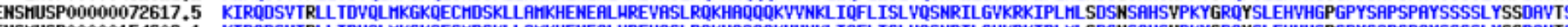

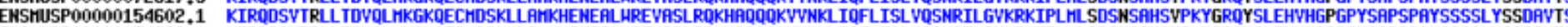

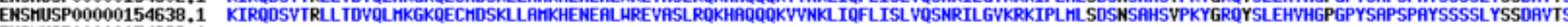
ENSHUSP00000154386.1

Consensus

kirqdsvt.11tdvqlnkgkqecndskllankhenealurevaslrqkhaqqqkvvnkliqflislvqsnrilgvkrkiplnl.ds,sahs.pky.rq.slehvhg.\&pysapspayssssly..dav.s $\begin{array}{llllllllllll}261 & 270 & 280 & 290 & 300 & 310 & 320 & 330 & 340 & 350 & 360 & 370\end{array}$

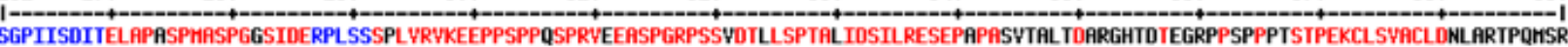

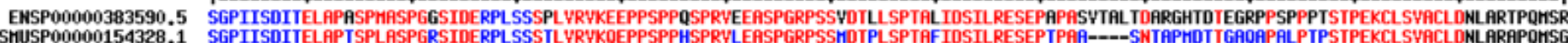
ENSP00000431512.1 ENSHUSP00000072617.5 ENSHUSP00000154602,1 ENSHUSP00000154638.1 Consensus

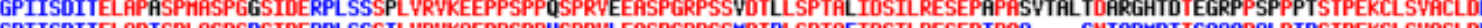
S

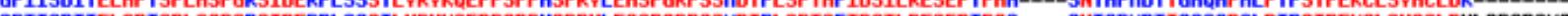
SGPIISDITELAPTSPLASPGRSIDERPLSSSTLVRVKQEPPSPPHSPRVLERSPGRPSSHOTPLSPTAFIOSILRESEPTPAR----SNTAPMDTTGAQAPALPTPSTPEKCLSVACLDNLARAPQHSE XELAPTSPLASPGRSIDE----STLVRVKGEPPSPPHSPRVLERSPGRPSSMTPLSPTAFIDSTLRESEPTPAR----SNTAPHDTTGAQPPALPTPSTPEKCLSVACLDK-

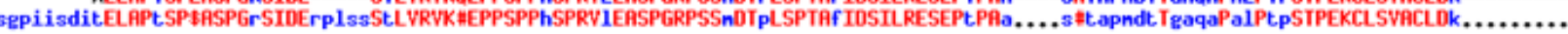

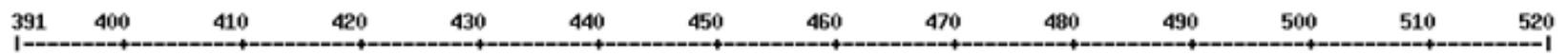

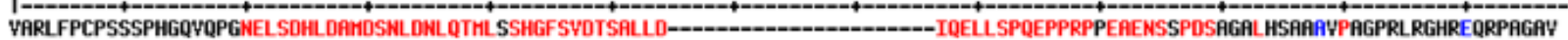

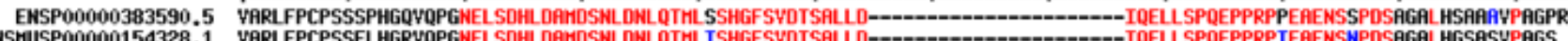

ENSHUSP00000154328.1

EHSPP0000431512.1

EHStusp00000154602.1

ENSHUSP00000154638.1

ENSHUSP00000154386,1

Consensus

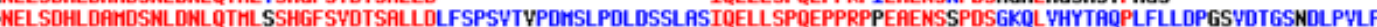
-NELSDHLDAMDSNLDNLOTHLTSHGFSYDTSALLD----10

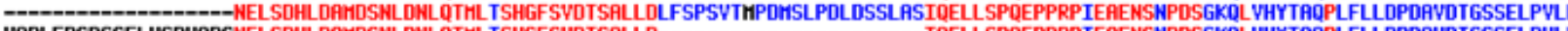
VARLFPCPSSFLHGRVQPGNELSDHLDAMOSNLDNLOTHLTSHGFSVDTSALLD-

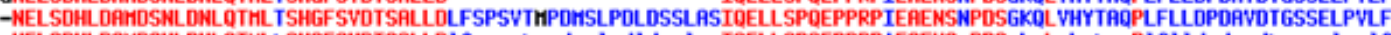
...................

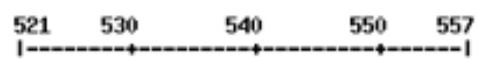

ENSP00000383590.5

ENSHUSP00000154328,

ENSP00000431512.1 ELGEGSYFSEGDGFAEDPISLLTGSEPPKAKDPTYS

ENSHUSP00000072617.5 ELGESSYFSEGDOYTDDPTISLLTGTEPHKAKDPTYS

ENSHUSP00000154602,1 ELGESSYFSEGDOYTDDPTISUIGTEPHKAKDPTVS

ENSHUSP00000154638.1 ELGESSYFSEGDOYTDDPTISLLTGTEPHKAKDPTVS

ENSHUSP00000154386.1 ELG

Consensus

\section{Figure 2}

Multiple sequence alignment of HSF1 


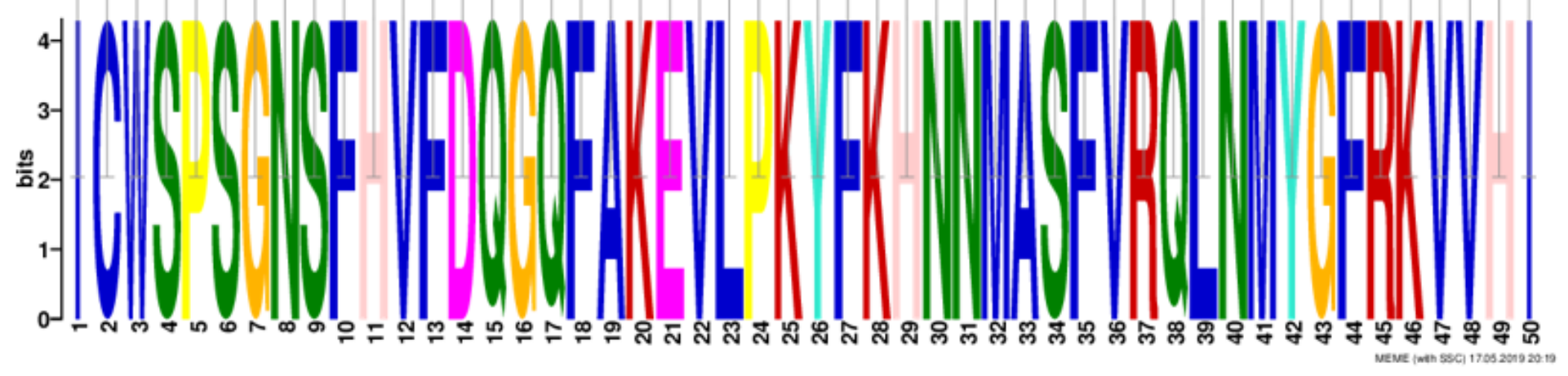

(a)

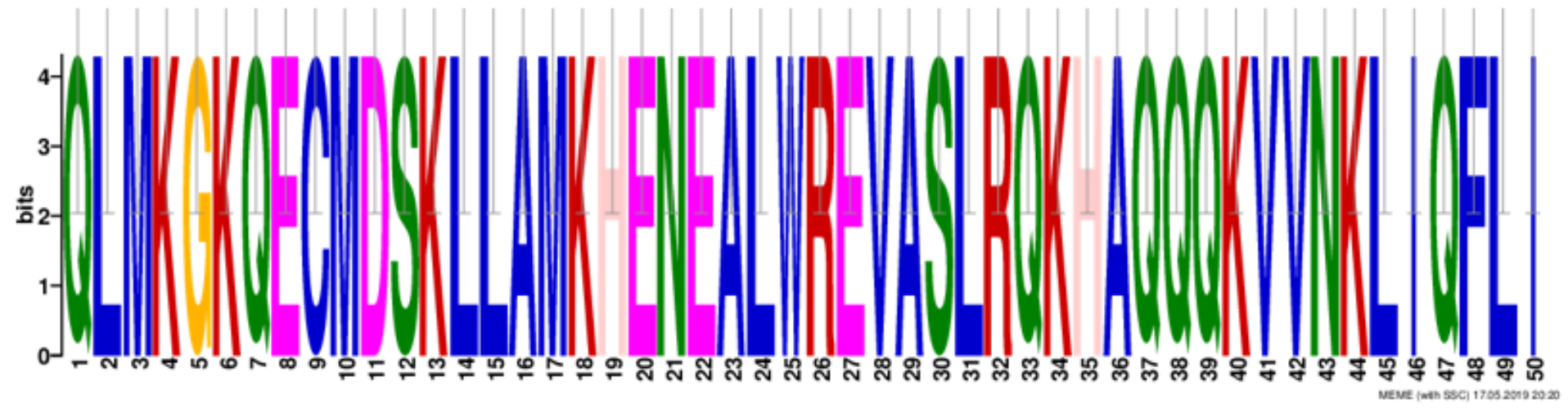

(b)

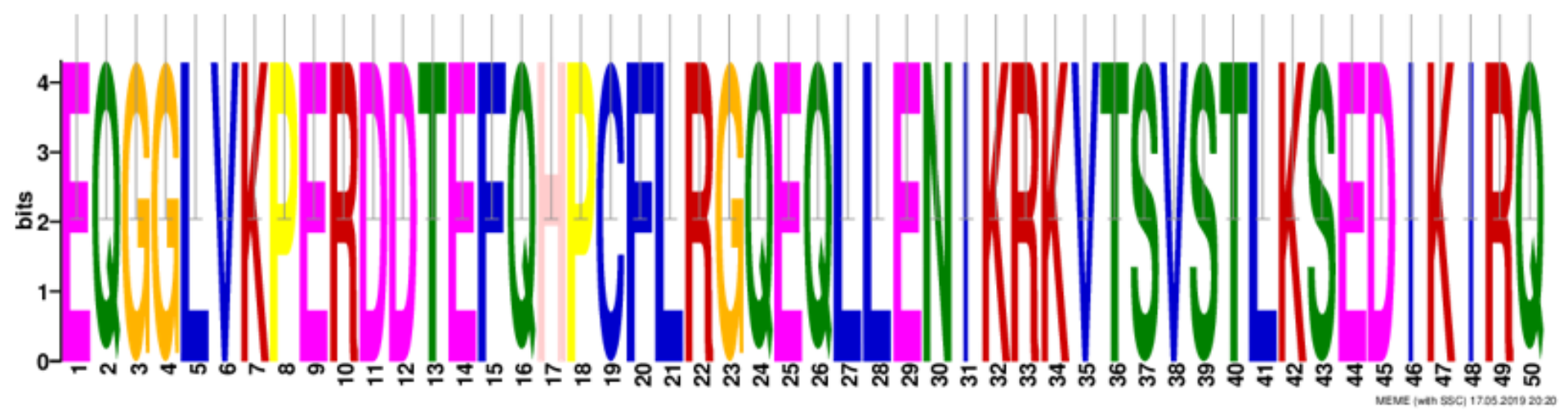

(c)

Figure 3

Specific motifs of HSF1 


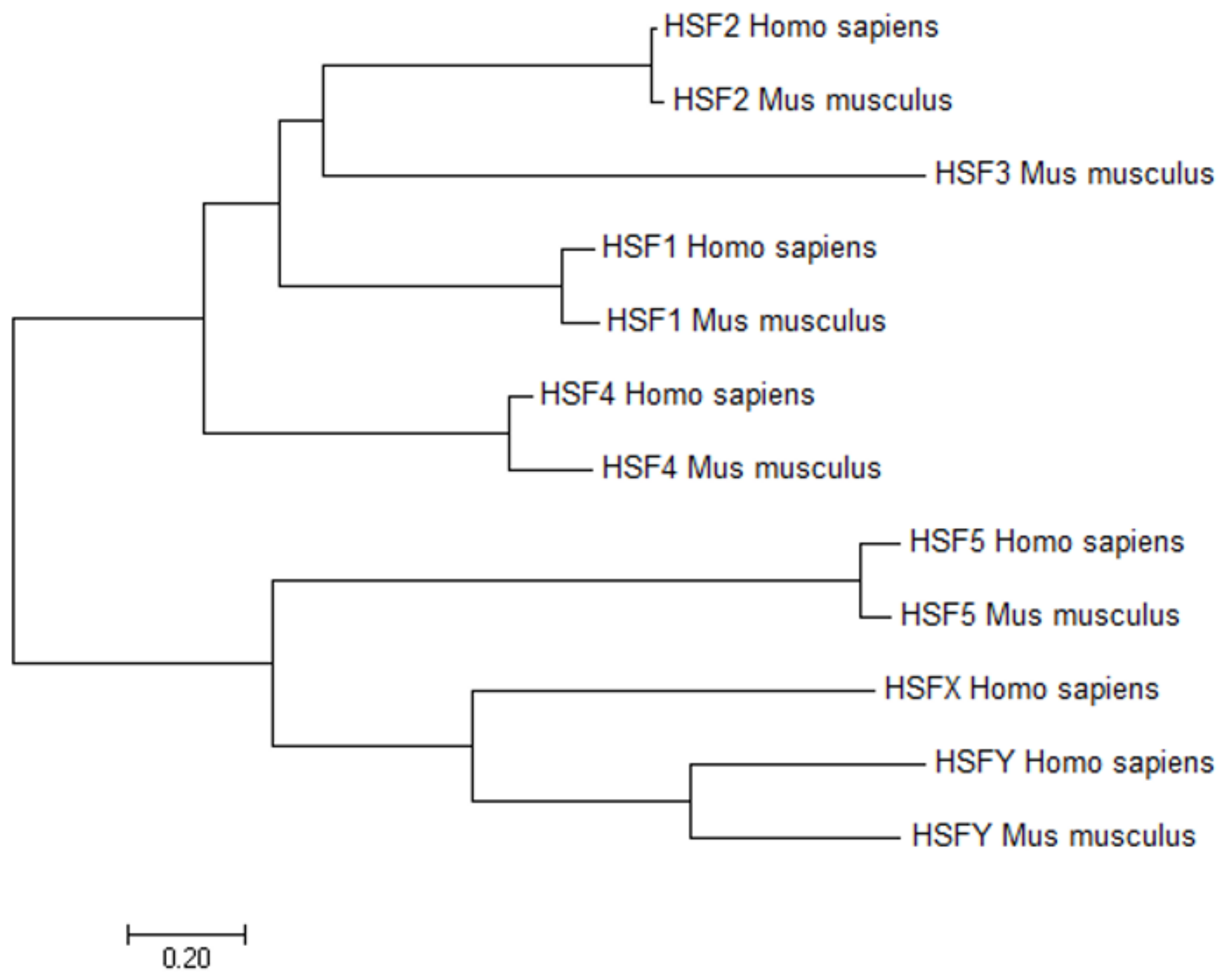

Figure 4

Phylogeny analysis of the HSF family 


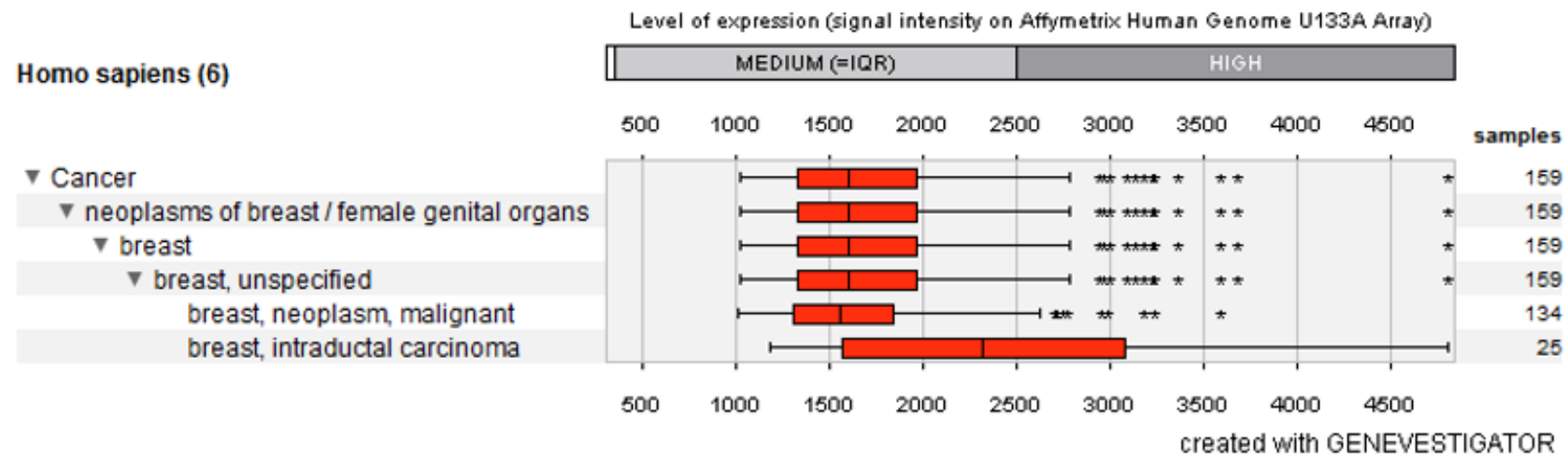

(a)

Dataset: 6 anat./neopl./cell. categories from data selection: DATA-HS_AFFY_U133A-7 Showing 1 measure(s) of 1 gene(s) on selection: HS-0

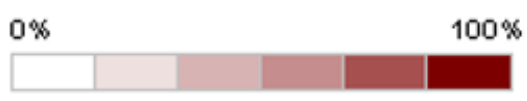

Percent of Expression Potential

Homo sapiens (6)

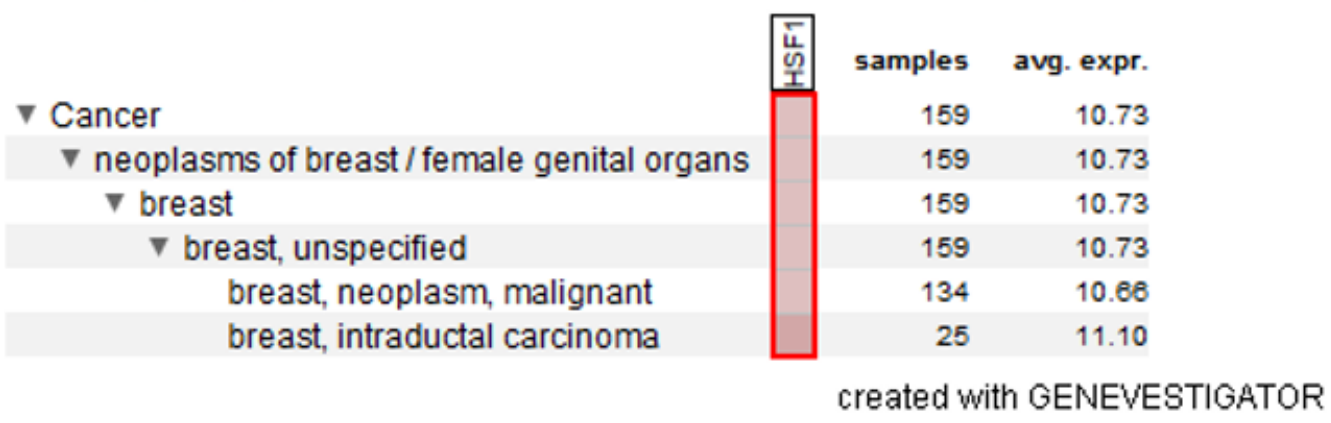

(b)

Figure 5

HSF1 expression in Breast Cancer 


\section{Homo sapiens (5)}

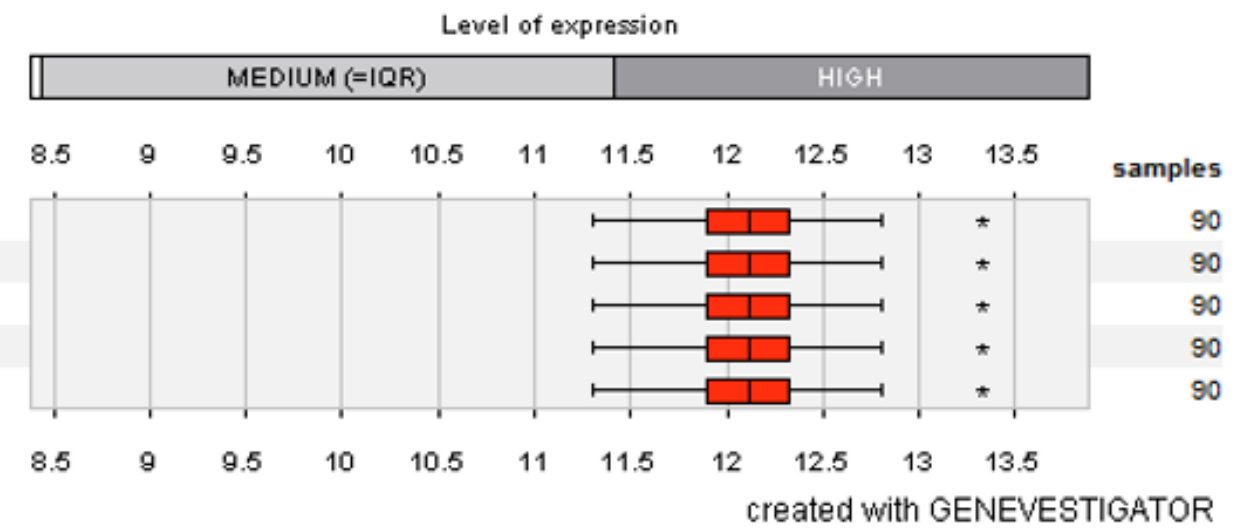

(a)

Dataset: 5 anat./neopl./cell. categories from data selection: DATA-HS_AFFY_U133PLUS_2-6 Showing 1 measure(s) of 1 gene(s) on selection: HS-0

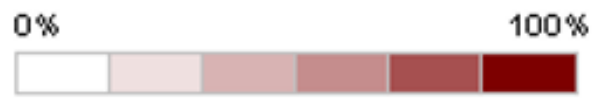

Percent of Expression Potential

\section{Homo sapiens (5)}

$\checkmark$ Cancer

$\checkmark$ neoplasms of digestive organs

$\checkmark$ colon

$\checkmark$ colon, unspecified colon, carcinoma, NOS

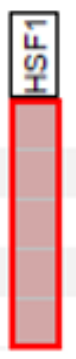

$\begin{array}{rr}\text { samples } & \text { avg. expr. } \\ 90 & 12.14 \\ 90 & 12.14 \\ 90 & 12.14 \\ 90 & 12.14 \\ 90 & 12.14\end{array}$

created with GENEVESTIGATOR

(b)

\section{Figure 6}

HSF1 expression in Colon Cancer 
Dataset: 11 anat/neopl/cell. categories from data selection: DATA-HS_mRNASeq_HUMAN_GL-5

Showing 1 measure(s) of 1 gene(s) on selection: HS-0

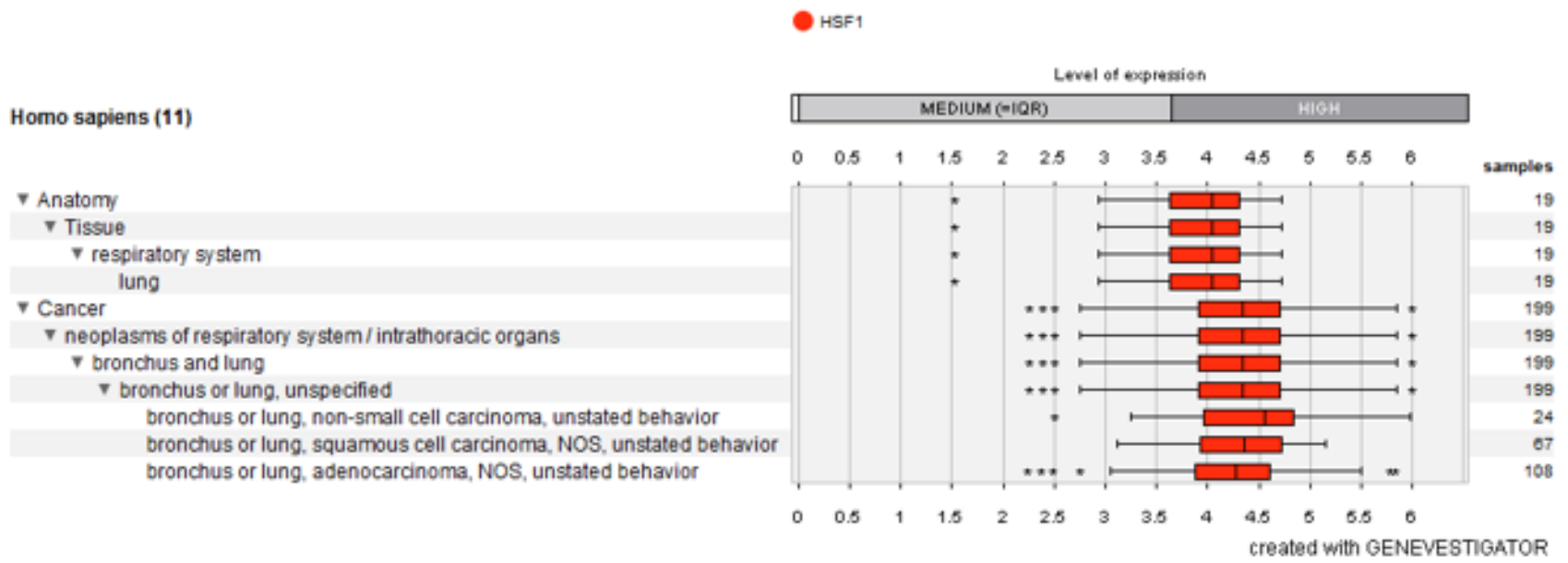

(a)

Dataset: 11 anat./neopl./cell. categories from data selection: DATA-HS_mRNASeq_HUMAN_GL-5

Showing 1 measure(s) of 1 gene(s) on selection: HS-0

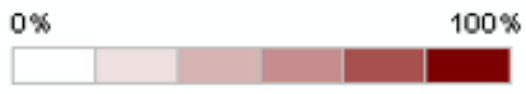

Percent of Expression Potential

\section{Homo sapiens (11)}

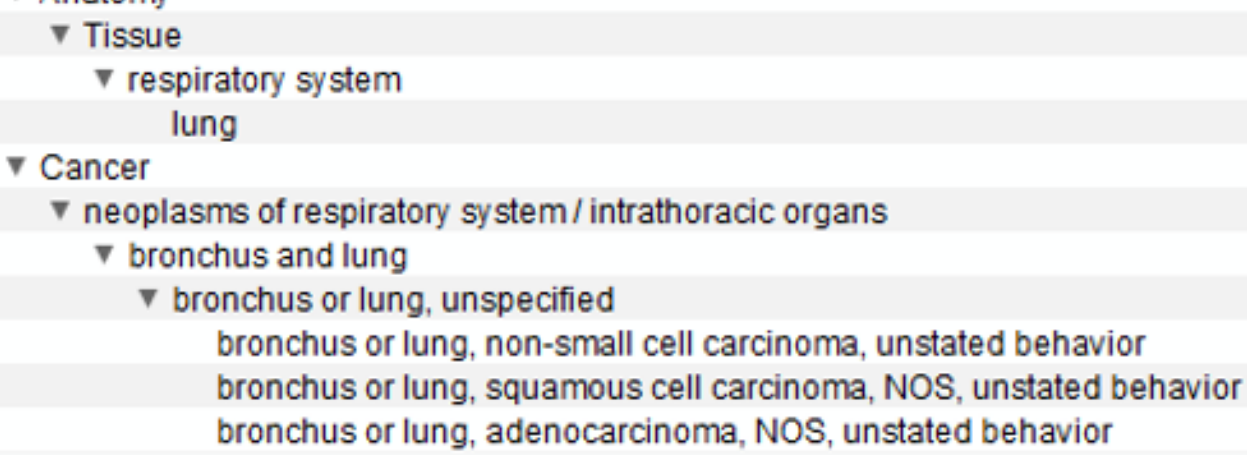

\begin{tabular}{|r|r|}
\hline 荝 \\
\hline samples & avg. expr. \\
19 & 3.87 \\
19 & 3.87 \\
19 & 3.87 \\
19 & 3.87 \\
199 & 4.27 \\
199 & 4.27 \\
199 & 4.27 \\
199 & 4.27 \\
24 & 4.43 \\
67 & 4.27 \\
108 & 4.23
\end{tabular}

created with GENEVESTIGATOR

\section{(b)}

\section{Figure 7}

HSF1 expression in Lung Cancer 
Chr 8

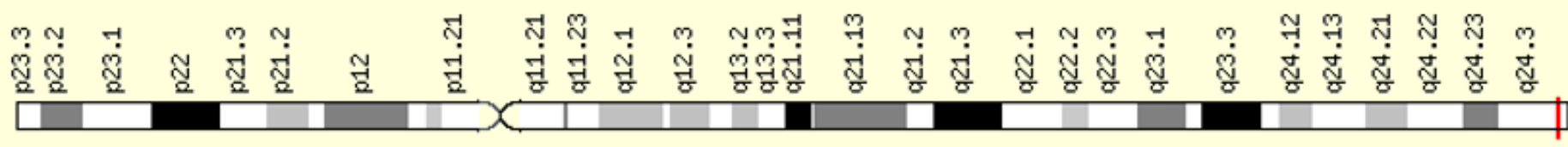

Figure 8

Chromosome location of HSF1

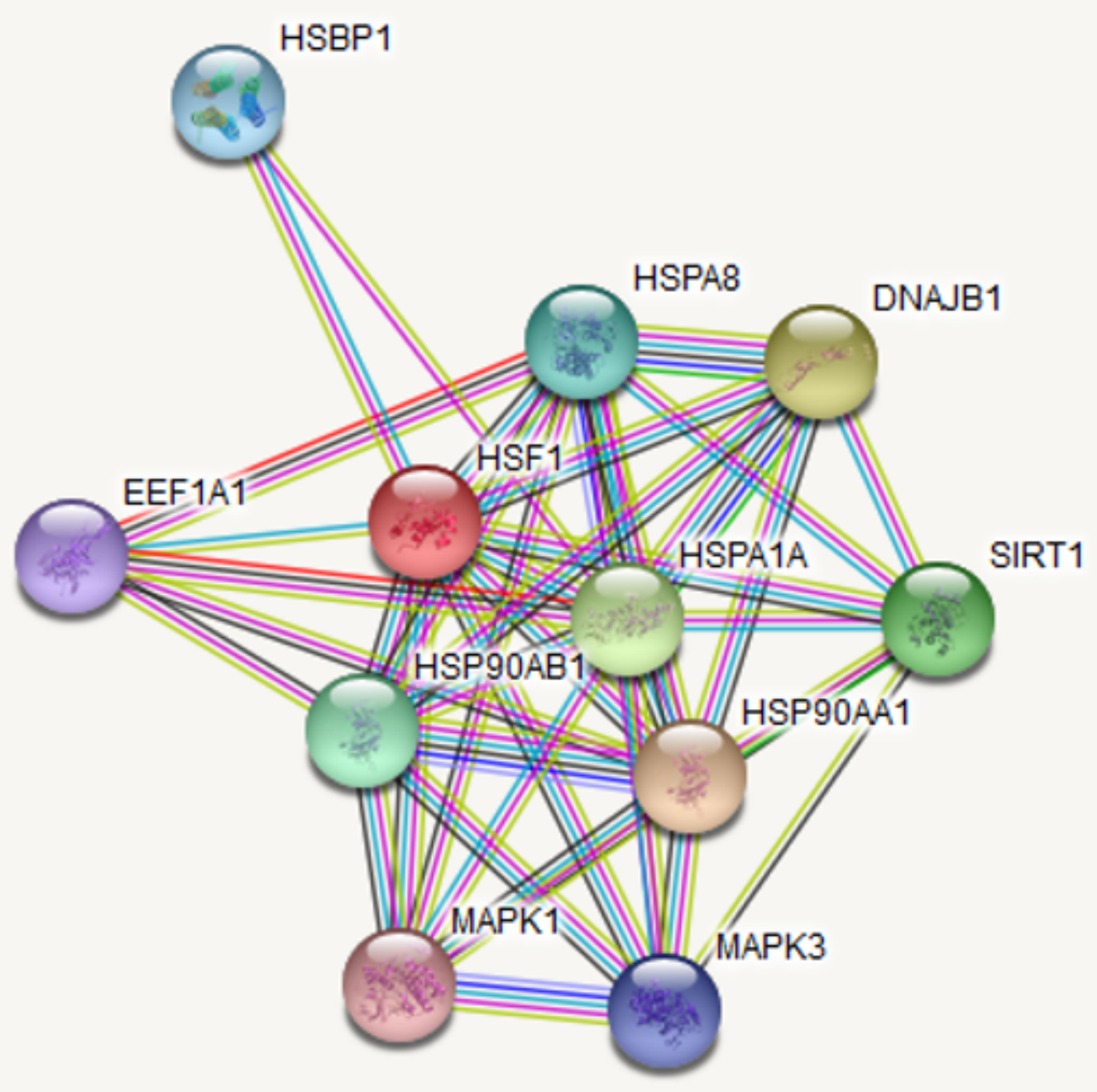

Figure 9

Gene regulatory network

\section{Supplementary Files}


This is a list of supplementary files associated with this preprint. Click to download.

- Table.doc 